\title{
LOCALIZACIÓN INDUSTRIAL EN EL MEDIO RURAL EN ARAGÓN. APROXIMACIÓN A SU CORRELACIÓN CON LAS EMPRESAS DE TRANSPORTE REGULAR Y DE MERCANCÍAS
}

\author{
José GARRIDO PALACIOS \\ Departamento de Geografía y Ordenación del Territorio \\ Universidad de Zaragoza
}

\begin{abstract}
Resumen: En este trabajo se estudia la localización industrial en el medio rural del ámbito aragonés, así como la correlación con las empresas de transporte regular y las agencias de transporte de mercancías.
\end{abstract}

Palabras clave: Localización industrial, medio rural, Aragón, empresas de transporte regular y agencias de transporte de mercancías.

\begin{abstract}
This work estudies the industrial location in the rural areas in Aragón and the correlation with the transport passenger companies and the freight transport agencies.
\end{abstract}

Key words: Industrial location, rural areas, Aragón, transport passenger company and freight transport agencies.

\section{OBJETIVOS Y METODOLOGÍA}

El objetivo de este estudio es conocer la localización de la industria en el medio rural correspondiente al ámbito aragonés y establecer las correlaciones con el asentamiento de las empresas de transporte regular y las agencias de transporte de mercancías.

Para ello, la metodología aplicada consiste en explicar el contexto de la industria en el medio rural con carácter general, así como los factores e hitos importantes que han jalonado el proceso industrial en Aragón. Asimismo se analiza la localización del número de empresas y trabajadores empleados en la industria y su correlación con las empresas de transporte regular y de mercancías. 
El criterio aplicado para la designación de medio rural es el conjunto de municipios inferiores a 10.000 habitantes, o sea, la población de hecho correspondiente al Censo de 1991 con la tipificación, según el INE, de rural (menos de 2.000 habitantes) y entre lo rural y lo urbano (entre 2.000 y 10.000 habitantes). El resultado de todo ello es la inclusión en el concepto de rural a todos los municipios aragoneses excepto aquellos que superan el umbral de los 10.000 habitantes; es decir, excluyendo los núcleos de Barbastro, Fraga, Huesca, Monzón, Alcañiz, Teruel, Calatayud, Ejea, Tarazona y Zaragoza.

\section{LA INDUSTRIA EN EL MEDIO RURAL}

La industria constituye una actividad económica que ha provocado mutaciones estructurales importantes en el medio rural. La reducción de la población activa empleada en la agricultura se ha compensado con el incremento de trabajadores en el sector secundario y los servicios. La localización fabril se integra en el paisaje rural y deja de ser patrimonio exclusivo del medio urbano. El agricultor-ganadero tradicional es sustituido paulatinamente por el obrero industrial, produciéndose una ruptura del binomio rural-agrario que antaño eran sinónimos.

Ahora bien, el interés que suscitan las actividades transformadoras en el medio rural en tiempos recientes no deben obviar su secular vinculación. En efecto, algunos de los rasgos específicos de la artesanía rural perviven en tiempos contemporáneos, siendo un buen ejemplo la localización industrial en núcleos de escasa población nutridos por la fuerza de trabajo excedentaria de la agricultura y apoyados en la existencia de recursos naturales y agrarios aptos para su transformación.

Más todavía, la localización fabril se ha concentrado en algunos casos en los valles para aprovechar la energía hidráulica obtenida de los cursos fluviales o la utilización del agua para actividades complementarias. Este es el ejemplo de buena parte de la localización de la industria algodonera, papelera, química, etc.

Otro factor de localización industrial en el espacio rural es el proceso difusor y de descentralización productiva de las empresas radicadas en las ciudades. El proceso de difusión supone la implantación industrial en espacios rurales, alejadas de zonas saturadas y elevadas deseconomías para las empresas: precio elevado del suelo, altos salarios, conflictividad laboral, congestión del tráfico, etc.

En este sentido, CUADRADO ROURA (1992) postula que las economías de escala están siendo sustituidas por economías de campo y unos métodos de producción más flexibles. 
Por otro lado, la descentralización trata, desde el punto de vista locacional, la ubicación de espacios periféricos (áreas rurales, regiones atrasadas, etc.) para reducir capacidad, mediante la subcontratación de pequeñas empresas, o de tareas, cuando se trata de operaciones de difícil o costosa mecanización/automatización. En la misma línea de la descentralización, LABORIE Y LAGUMIER (1982) afirman la existencia de una jerarquización del espacio desde el interior de las ciudades a las áreas rurales más alejadas.

En cuanto a la estructura industrial en el medio rural, los rasgos comunes más significativos son: el predominio de la pequeña empresa y la utilización de tecnologías intermedias, sin olvidar la inversión exterior que, a veces, se materializa con la implantación de grandes empresas (MOLINERO, 1990).

\section{LA INDUSTRIA EN ARAGÓN}

Las características actuales de la industria en la Comunidad Autónoma de Aragón son consecuencia de factores complejos gestados desde tiempos pretéritos. Así pues, la desvinculación entre el proceso industrial y el aprovechamiento de los recursos existentes en el territorio y la acusada dependencia del exterior son algunos de esos factores condicionantes (MOLINA Y MARIN, 1981).

Si bien el artesanado regional constituyó la base de la transformación industrial en otras regiones, en Aragón apenas ha tenido relevencia para los actuales procesos de producción. De hecho, la localización del artesanado tradicional estuvo disperso por todo el territorio, mientras que en la actualidad no coincide, grosso modo, con los grandes focos industriales de Aragón. De esta regla general constituye una digna excepción el caso de los núcleos de Illueca y Brea, los cuales han evolucionado de una actividad de curtido de pieles a la fabricación de calzado en la actualidad.

Por otro lado, la emergencia de los primeros centros industriales están ligados fundamentalmente a capital extrarregional. Buenos ejemplos de ello se encuentran en la afluencia de capital vasco a principios del siglo XX para la explotación de yacimientos de hierro en Ojos Negros (Teruel), el capital de Banco Urquijo para la industria química instalada en Sabiñanigo y la entrada del grupo francés Pechiney para la instalación de "Aluminio Español" (CLIMENT Y ALONSO, 1995).

En cualquier caso, Aragón no puede considerarse como región industrial hasta 1975, que es el momento cuando la población activa empleada en el sector secundario supera al sector agrario. De ahí se deriva, por tanto, que el proceso de industrialización de la Comunidad aragonesa es tardío, no sólo respecto a los países 
del Norte de Europa, pioneros de la Revolución Industrial, sino frente a sus regiones vecinas como Cataluña y País Vasco, que iniciaron este proceso con varias décadas de antelación.

Ahora bien, pese a su incorporación, el camino está jalonado por una serie de hitos que han impulsado ese proceso industrial en Aragón; estos son los de la producción de azúcar, el Polo de Desarrollo de Zaragoza y la implantación de la General Motors, actual Opel España.

La industria de la remolacha fue la actividad impulsora a finales del siglo XIX y principios del $X X$, estando localizada esencialmente en la alineación de Zaragoza con el País Vasco y Madrid; es decir, en las proximidades del trazado del ferrocarril ${ }^{1}$, pues en el corredor o eje ${ }^{2}$ del Ebro se encontraban las azucareras de Casetas, Alagón y Gallur (Climent y Alonso, 1995), mientras que en el eje del Jalón se ubican diversas fábricas en Epila, Calatayud, etc. Posteriormente se establecen fábricas en otros puntos del territorio aragonés, tales como en los valles del Jiloca y Martín (Teruel) y en e1 valle del Cinca (Huesca), de modo que potenciaron esta industria. De todas ellas es importante destacar el carácter rural, en su mayor parte, bien por motivos de proximidad, bien por su cercanía al ferrocarril.

El desarrollo del Polo industrial entre 1964 y 1969 fue otro factor importante en el proceso del sector secundario. Su implantación posibilita la instalación de 174 empresas, si bien la mayoría estaban previamente en Zaragoza. El mayor impulso lo experimentaron las industrias de papel y transformados metálicos, lo que justifica de alguna manera la especialización productiva de Aragón. Desde el punto de vista locacional, el Polo de Desarrollo propició la concentración de la industria aragonesa y la polarización, con la consiguiente atracción de capitales y personas hacia ese Polo.

Finalmente, la implantación de General Motors en los años ochenta en el mencionado corredor del Ebro ha potenciado la concentración industrial en esa zona, pues el peso de la industria automovilística rebasa el ámbito local para convertirse en el principal motor de la industria aragonesa, que difunde sus efectos a un amplio territorio. En efecto, parte de sus industrias auxiliares se localizan en Tarazona, Fuentes de Ebro, Belchite, ... e incluso Logroño. Así pues, tanto por su volumen de empleo (más de 9000 trabajadores) como por los efectos inducidos espaciales y

\footnotetext{
I El ferrocarril constituye uno de los factores clave del despegue industrial y actúa como factor de localización de otros establecimientos industriales. Aunque, por otra parte, con el ferrocarril se pretendia más la relación de tipo económico que la conexión interregional para armonizar el territorio nacional (Molina y M arín, 1981).

2 El término de "Eje" está definido por Calvo Palacios en "El Eje de desarrollo del Valle del Ebro y las perspectivas del Eje Norte-Sur", 3" Congreso de Economía Aragonesa, Zaragoza (1994).
} 
económicos que genera, la empresa Opel España actual constituye el punto de referencia principal de la industria aragonesa.

Pues bien, la ubicación en el término municipal de Figueruelas refuerza el carácter rural de su instalación y explica las ventajas de poseer unas buenas comunicaciones y el efecto "periurbano" de la capital zaragozana, atrayendo otras industrias con su política de abastecimientos "just in time" que condiciona la localización próxima de empresas auxiliares (ALONSO, 1993).

En suma, la industria en Aragón pone de relieve el proceso tardío de su desarrollo y una localización concentrada en las alineaciones que conducen a Zaragoza y a relativa poca distancia de la capital.

\section{LOCALIZACIÓN INDUSTRIAL EN EL MEDIO RURAL}

Se ha comentado anteriormente que el proceso industrial en Aragón tiene una especial incidencia en el medio rural y en el entorno de las ciudades. Pues bien, en principio observaremos cómo la mayoría de las empresas y de los trabajadores empleados en la industria en Aragón tienen en común precisamente ese carácter rural.

Tabla 1.- Indicadores de Desarrollo Industrial y Población Fuente: INE. Censo Industrial (1993) y Censo de Población (1991). Elaboración propia.

\begin{tabular}{|c|c|c|c|}
\hline & NEMPRESAS & $N^{\circ}$ EMPLEADOS INDUSTRIA & POBLACION \\
\hline PROV.HUESCA & 2156 & 26640 & 218897 \\
\hline Medio Rural & 1612 & 18753 & 112573 \\
\hline$\%$ Rural & 74,76 & 70,4 & 51,43 \\
\hline PROV. ZARAGOZA & 9928 & 174826 & 861329 \\
\hline Medio Rural & 7142 & 124312 & 194546 \\
\hline$\%$ Rural & 71,93 & 71,1 & 22,58 \\
\hline PROV. TERUEL & 1668 & 20246 & 141320 \\
\hline Medio Rural & 1430 & 16819 & 97432 \\
\hline$\%$ Rural & 85,79 & 83,9 & 68,94 \\
\hline ARAGÓN & 13752 & 221512 & 1221546 \\
\hline Medio Rural & 10185 & 159889 & 404551 \\
\hline$\%$ Rural & 74,06 & 72,17 & 33,11 \\
\hline
\end{tabular}


En cuanto al número de empresas industriales en Aragón, la mayor parte de ellas se localizan en el medio rural $(74 \%)$, lo que corrobora el interés fabril por situarse fuera de las ciudades. Por su parte, la provincia de Teruel es la de localización más rural del conjunto aragonés, pues frente al $85.79 \%$ de esta provincia se opone el $71.93 \%$ de la provincia de Zaragoza. Esto obedece a que el número de ciudades es mayor en la última provincia que en Teruel, lo que determina las posibles ubicaciones industriales.

Si se considera el número de empleados industriales, los valores se aproximan al número de empresas, lo cual señala que, independientemente del tamaño de la empresa, los trabajadores ejercen su función profesional en el medio rural.

Por otro lado, si se comparan estos indicadores de la industria con la residencia de la población de hecho en 1991, se deriva un claro contraste en el conjunto de la Comunidad aragonesa, ya que sólo el $33.11 \%$ de los habitantes residen en el medio rural, siendo muy acusada la diferencia entre la provincia de Zaragoza $(22,58 \%)$ y la provincia de Teruel $(68.94 \%)$.

De todo ello se pueden deducir varias cuestiones: primera, la población que reside en el medio rural supone un tercio de la población total de Aragón; segunda, los trabajadores prefieren residir en la ciudad mientras que su trabajo en la industria está localizado fuera de los municipios urbanos; tercera, la provincia "más urbana" es la de Zaragoza, si bien la "más rural" es la de Teruel; cuarto, en la provincia de Zaragoza se requieren más medios de transporte para trasladarse a su trabajo en función del reducido número de habitantes que residen en el medio rural; y quinta, la provincia de Teruel es la más equilibrada desde el punto de vista de los lugares de residencia y de trabajo en la industria.

El mapa de localización de empresas industriales asentadas en el medio rural. muestra los municipios donde la concentración es más acusada. Así, en la provincia de Huesca se ubican esencialmente en la zona del Cinca, como Binéfar (61 empresas) y Tamarite (14 empresas), así como en el municipio de Sabiñanigo (29 empresas), aunque este enclave carece de continuidad en su entorno. Igualmente sucede en la provincia de Teruel, dado que si bien en la zona del Bajo Aragón existe una nítida concentración empresarial (Calanda, 38 empresas; Andorra, 24; y Alcorisa, 22), en el resto del territorio aparecen enclaves perdidos en el desierto industrial; este es el caso de Calamocha (29 empresas) y Mora de Rubielos (20 empresas).

Más definido se encuentra el panorama locacional de la provincia de Zaragoza, donde se ha generado una estructura radial en torno a la capital que permite establecer alineaciones o corredores coincidentes con al salida de la ciudad y que conectan con otras urbes, a saber: 
- Zaragoza-País Vasco. Es el corredor mejor definido de los expuestos, tanto por su volumen como por la continuidad de su implantación. En él se localizan los municipios de Utebo (128 empresas), Alagón (33 empresas), Figueruelas (14 empresas), Pedrola (14 empresas) y Pinseque (27 empresas).

- Zaragoza-Madrid. Constituye otro corredor de notable importancia en el entramado industrial de la región aragonesa. Se apoya en los núcleos de Epila (23 empresas) y La Almunia (36 empresas), aunque se puede incorporar también el municipio de Illueca (104 empresas), bañado por el río Aranda, afluente del Jalón.

- Zaragoza-Teruel. Es muy relevante, en particular la salida de la capital zaragozana, pues poseen gran peso industrial los núcleos de Cuarte de Huerva (404 empresas) y María de Huerva (43 empresas), y algo menos trascendencia fabril el de Cariñena (17 empresas).

- Zaragoza-Barcelona. Destacan los municipios de Quinto de Ebro (18 empresas) y Caspe (56 empresas), aunque el último se encuentra bastante alejado.

- Zaragoza-Huesca. Es interesante reseñar los municipios de Villanueva del Gállego (98 empresas) y Zuera (43 empresas).

En definitiva, se puede resumir lo anterior afirmando que la localización de la industria en el medio rural se concentra en dos corredores principales, definidos por los corredores fluviales del Ebro y Valle del Jalón, y el entomo próximo a Zaragoza, añadiendo las zonas del Bajo Aragón y del Cinca. El resto son enclaves periféricos que forman parte del entramado empresarial de Aragón, y que pueden servir de apoyo para impulsar futuros desarrollos en su área respectiva en aras de equilibrar el reparto espacial de la industria aragonesa.

Por otro lado, en el mapa de empleo industrial la situación no difiere en exceso de lo anterior. En principio, la localización del volumen de trabajadores en la industria se concentra, al igual que las empresas, en el entorno de Zaragoza con enclaves aislados en Sabiñanigo, Binéfar, Andorra, etc.

Sin embargo, existe un hecho diferencial en Figueruelas, donde un número de empresas limitado genera un elevado volumen de empleados y atrae asimismo otras empresas a su área de influencia. Este es el claro ejemplo de Opel España, pues con 9798 empleados ${ }^{3}$ representa el mayor centro de ocupación industrial de Aragón en el medio rural y ejerce un fuerte poder de atracción en puntos que traspasan las fronteras municipales (ALONSO, 1993).

\footnotetext{
${ }^{3}$ Según el Censo Industrial de 1993.
} 
MAPA No

N DE EMPRESAS INDUSTRIALES EN EL MEDIO RURAL (MÁS DE 5 EMPRESAS)

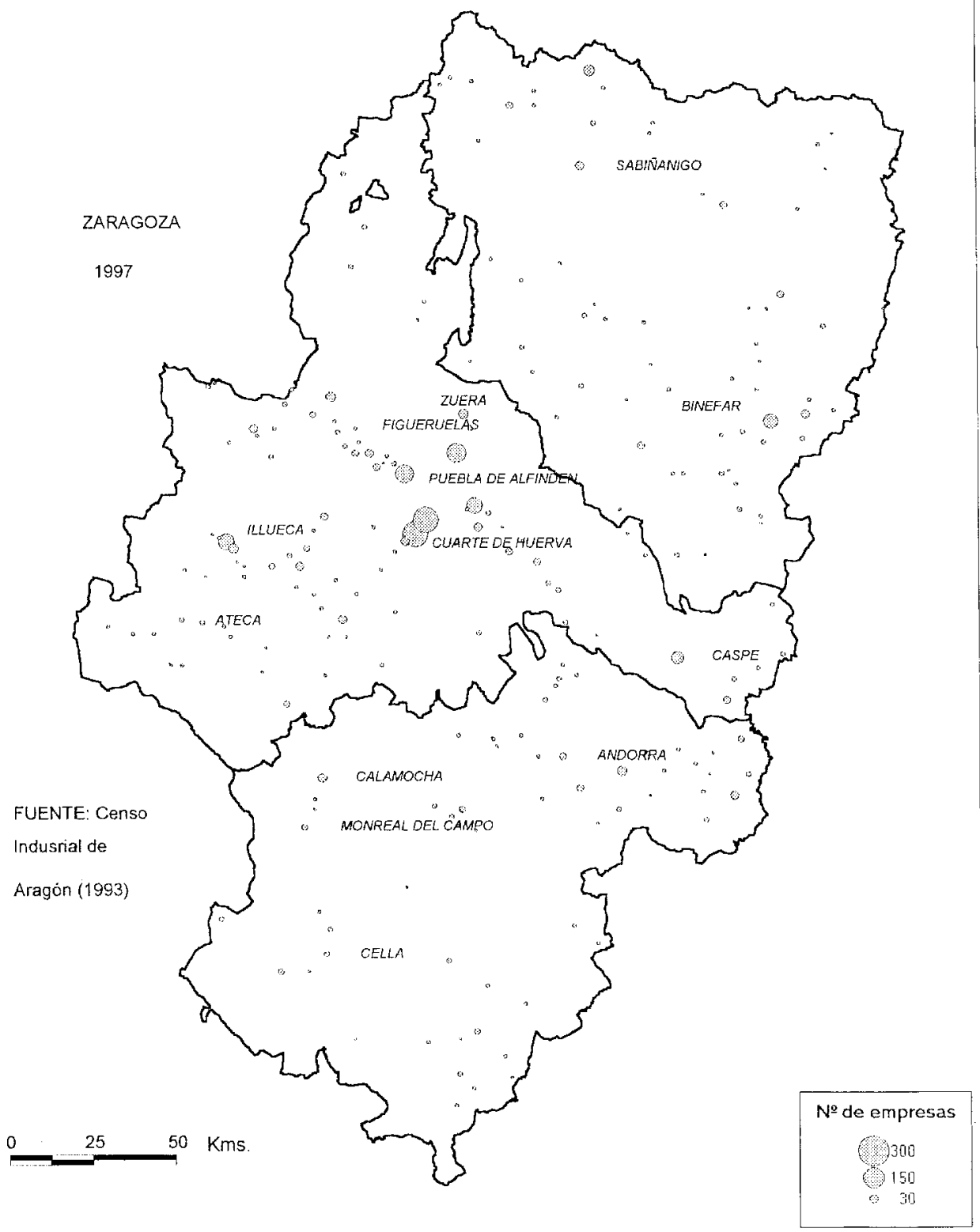


MAPA N $N^{\circ}$

EMPLEO INDUSTRIAL EN EL. MEDIO RURAL (MÁS DE 10 TRABAJADORES)

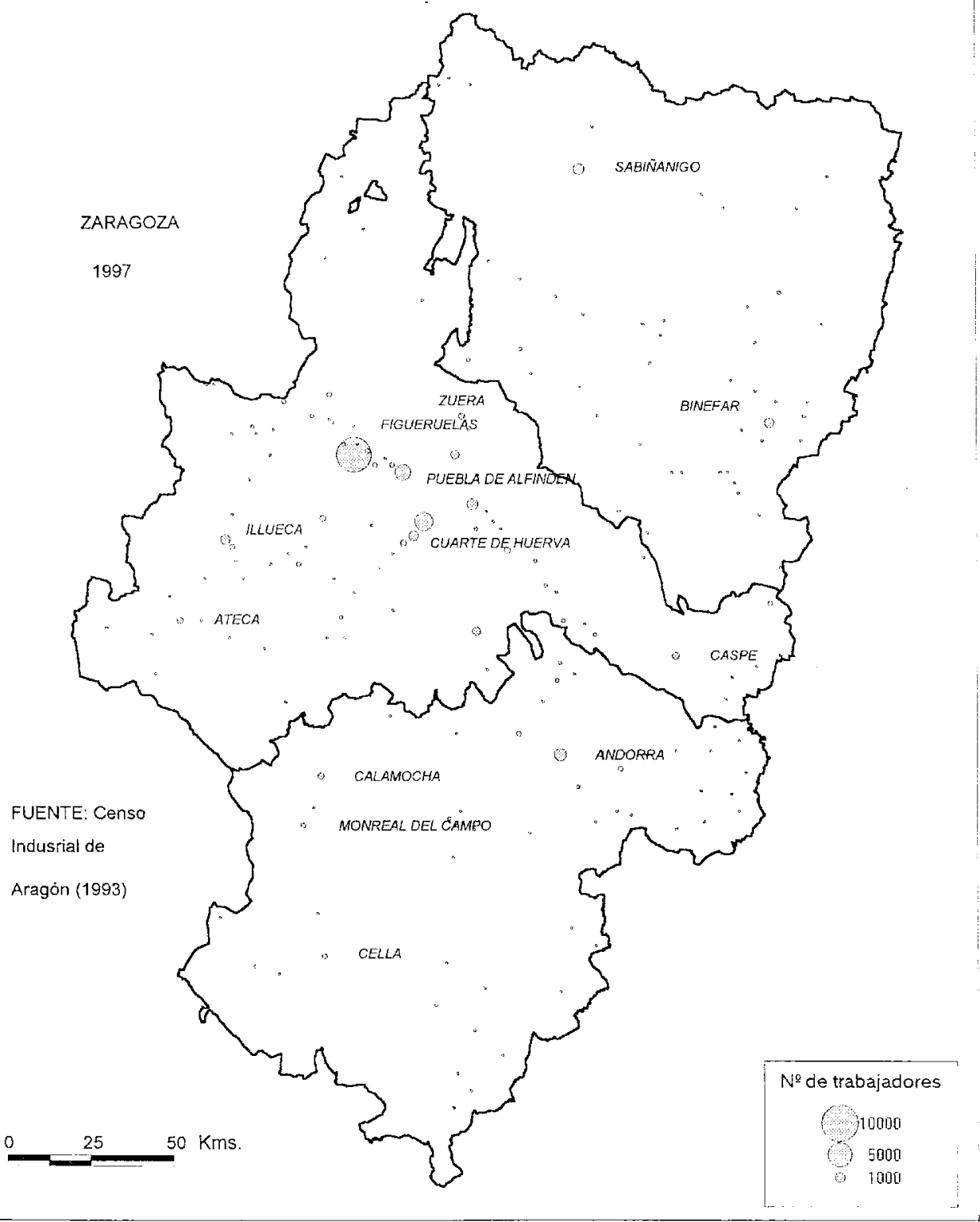


Por su parte, otros núcleos relevantes por el número de empleados son los de Cuarte de Huerva (4207 empleados), Utebo (2983 empleados), Illueca (1450 empleados), Villanueva del Gállego (1402 empleados), Sabiñanigo (1151 empleados), Binéfar (762 empleados), Andorra (522 empleados), etc.

En suma, el mayor volumen de trabajadores por municipio (superior a 1000 trabajadores) se localiza de nuevo en los corredores de acceso a Zaragoza y en los enclaves de Sabiñanigo e Illueca.

Por último, la dimensión media de las empresas en el medio rural aragonés es de carácter minifundista, pues cada empresa genera 15.7 trabajadores en al industria, cifra similar a la media de Aragón (16.10), lo que define una de las características del sector industrial aragonés. En general, no existe apenas diferencia entre la dimensión de la empresa y empleados en el medio rural o urbano, pero sí contrasta la media de la provincia de Teruel (11.7 empleados por empresa) y la provincia de Zaragoza (17.4 empleados por empresa), lo que explica la existencia de grandes empresas asentadas en ésta última entidad, como la Opel España mencionada, El Aguila en Utebo, etc.

\section{NIVEL DE ESPECIALIZACIÓN DE LA INDUSTRIA ARAGONESA}

La profundización en la industria aragonesa requiere conocer el grado de especialización de esta actividad. Así, en el Gráfico 1 se ofrece una visión de conjunto del tejido industrial integrando las diversas ramas industriales por agrupamientos de actividad.

En primer lugar, la industria argonesa presenta un perfil bastante diversificado, puesto que todas las agrupaciones tienen una relativa representación en el sector secundario, pero esa característica no oculta una clara especialización, donde, en función de los indicadores de personas ocupadas en la industria y Valor Añadido Bruto a coste de los factores, reflejan una mayor proporción de los subsectores de material de transporte; alimentación, bebidas y tabaco; maquinaria y equipo mecánico; metalurgia y productos metálicos; y, en último lugar, el textil, confección, cuero y calzado.

La agrupación de material de transporte sobresale del resto, pues absorbe el $22.91 \%$ del empleo industrial y el $15.76 \%$ del V.A.B. Por tanto, en Aragón esta actividad se considera muy especializada, ya que representa el $12.28 \%$ del V.A.B. de la producción nacional frente al $4.08 \%$ de la media de España. 
Gráfico 1.- Agrupaciones de actividad industrial (1993).

Fuente: D.G.A. Instituto Aragonés de Estadística. Cuentas de la industria aragonesa (1993).

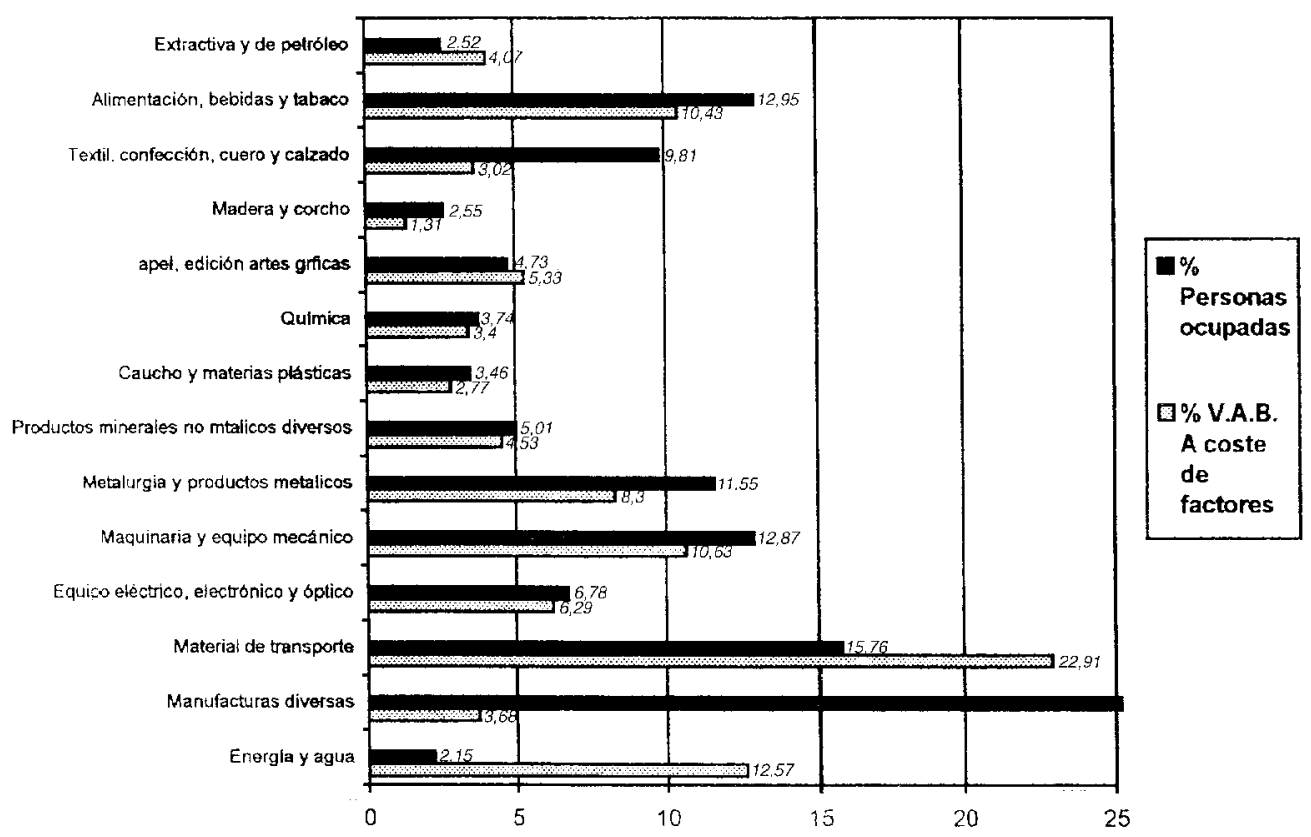

Comparando los porcentajes de alimentación, textil-calzado y madera de Aragón y España, la región no resulta especializada en ninguna de ellas, aunque tengan un peso significativo en el V.A.B. y empleo regionales.

Después de la rápida visión de las actividades industriales nos interesa principalmente la localización de las mismas y con especial relevancia aquellas que tienen mayor incidencia en la industria regional. En consecuencia, a continuación se pone el acento en el transporte como elemento de conexión de las relaciones económicas territoriales.

Nos centraremos en la localización de las empresas de transporte regular y el número de agencias de transporte de mercancías. Estos dos indicadores van a coadyuvar a comprender las relaciones empresa-empleados industriales y empresatransporte del producto. Todo ello en el entorno del medio rural donde se ubican las tres cuartas partes de las empresas y el empleo de este sector. 


\section{LOCALIZACIÓN DE LAS EMPRESAS DE TRANSPORTE REGULAR Y AGENCIAS DE TRANSPORTE DE MERCANCÍAS}

Los mapas 3 y 4 tratan de comprobar la posible correlación entre la localización industrial y las empresas que gestionan el desplazamiento de trabajadores y mercancías que generan las empresas. En ambos casos la información está incompleta a causa de que la mayoría de las sedes empresariales residen en las capitales provinciales. Por ejemplo, en la provincia de Zaragoza existen 24 empresas de transporte regular y 15 de ellas residen en la capital. Lo mismo ocurre en el resto de provincias, dado que el $50 \%$ de las empresas están ubicadas en sus capitales respectivas. Pese a ello, también es cierto que la necesidad de desplazarse al lugar de trabajo genera en sus proximidades efectos spread que favorecen otras actividades. Este es el caso que nos ocupa.

Las empresas de transporte regular tienen un carácter periférico en la Comunidad aragonesa, lo que parece que puede explicar la mayor necesidad de estos servicios por la ausencia de otro tipo de transporte: ferrocarril, carreteras de gran capacidad, etc. En segundo lugar, las empresas se asientan en zonas donde existen actividades industriales, tales como Tamarite de Litera, Caspe, Brea, Calamocha, etc. En tercer lugar, existen localizaciones próximas a centros industriales de cierta relevancia, como Añón (próximo a Tarazona), Cella (cercano a Teruel), Alcalá de la Selva (próximo a Mora de Rubielos), etc.

En resumen, el reparto espacial de este minifundismo empresarial, donde predomina la empresa unitaria, salvo en Cella que residen dos empresas, responde a la necesidad periférica de transporte del personal trabajador entre la localización de la industria y su residencia habitual. Recordemos en este sentido que las dos terceras partes de la población reside en los núcleos mayores de 10.000 habitantes.

Respecto al número de agencias de transporte de mercancías, la proporción entre las sedes urbanas y rurales está más distorsionada que en el caso anterior, pues de las 160 existentes en Aragón, 104 corresponden a Zaragoza capital, mientras que en Huesca y Teruel disponen de 7 y 6 agencias, respectivamente. Por tanto, la polarización de las agencias es más acusada en beneficio de Zaragoza.

Ahora bien, el resto de las sedes presentan una mayor centralidad, tanto en cuanto a la concentración en la provincia de Zaragoza como la ausencia de enclaves tan periféricos como las empresas de transporte regular. Además, la coincidencia es mayor cuanto más acusado es el número de empresas ubicadas. En efecto, la cuantía más elevada corresponde a Cuarte de Huerva, Tamarite-Monzón-Binéfar, Caspe, Calamocha, Andorra, etc. 


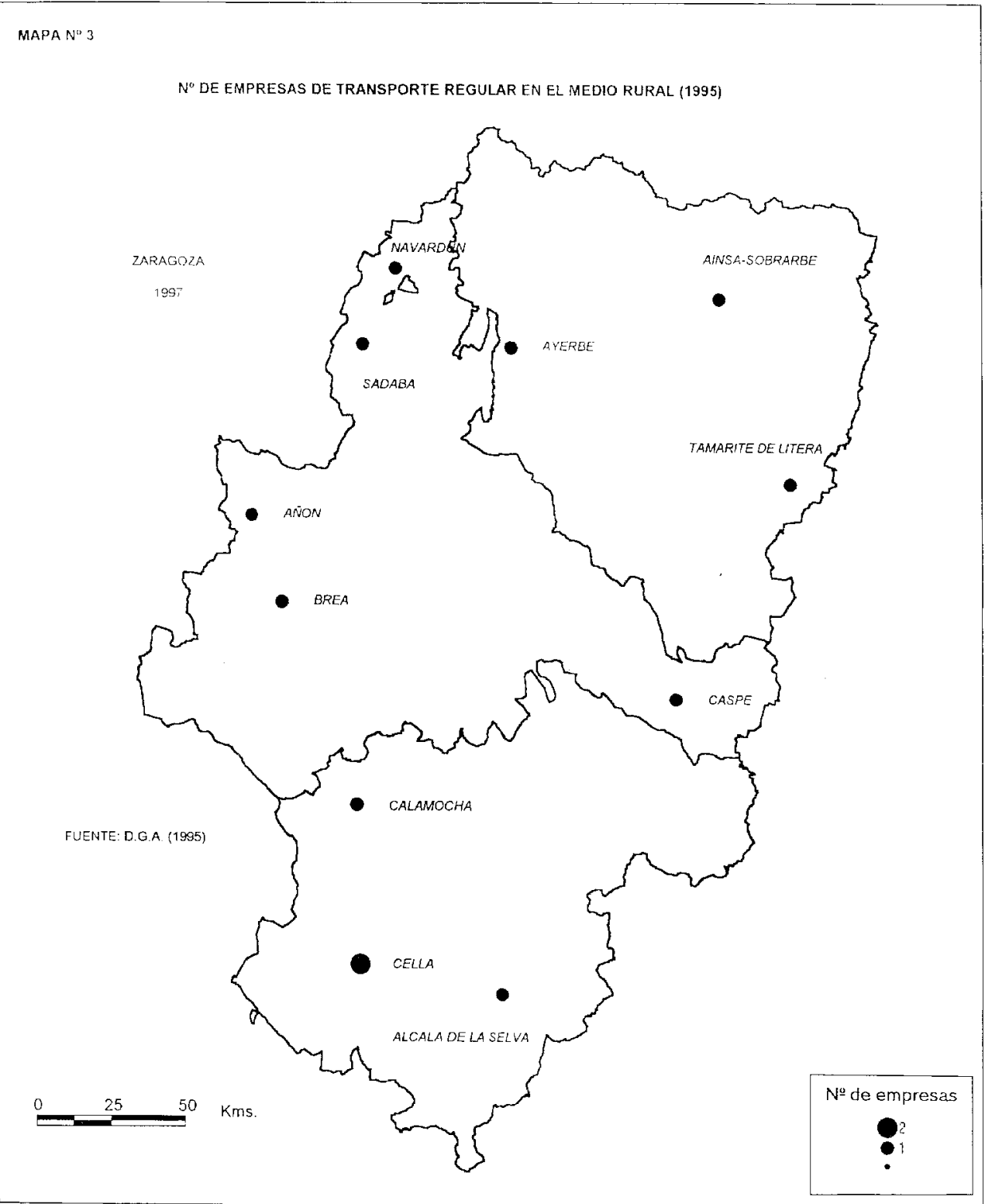




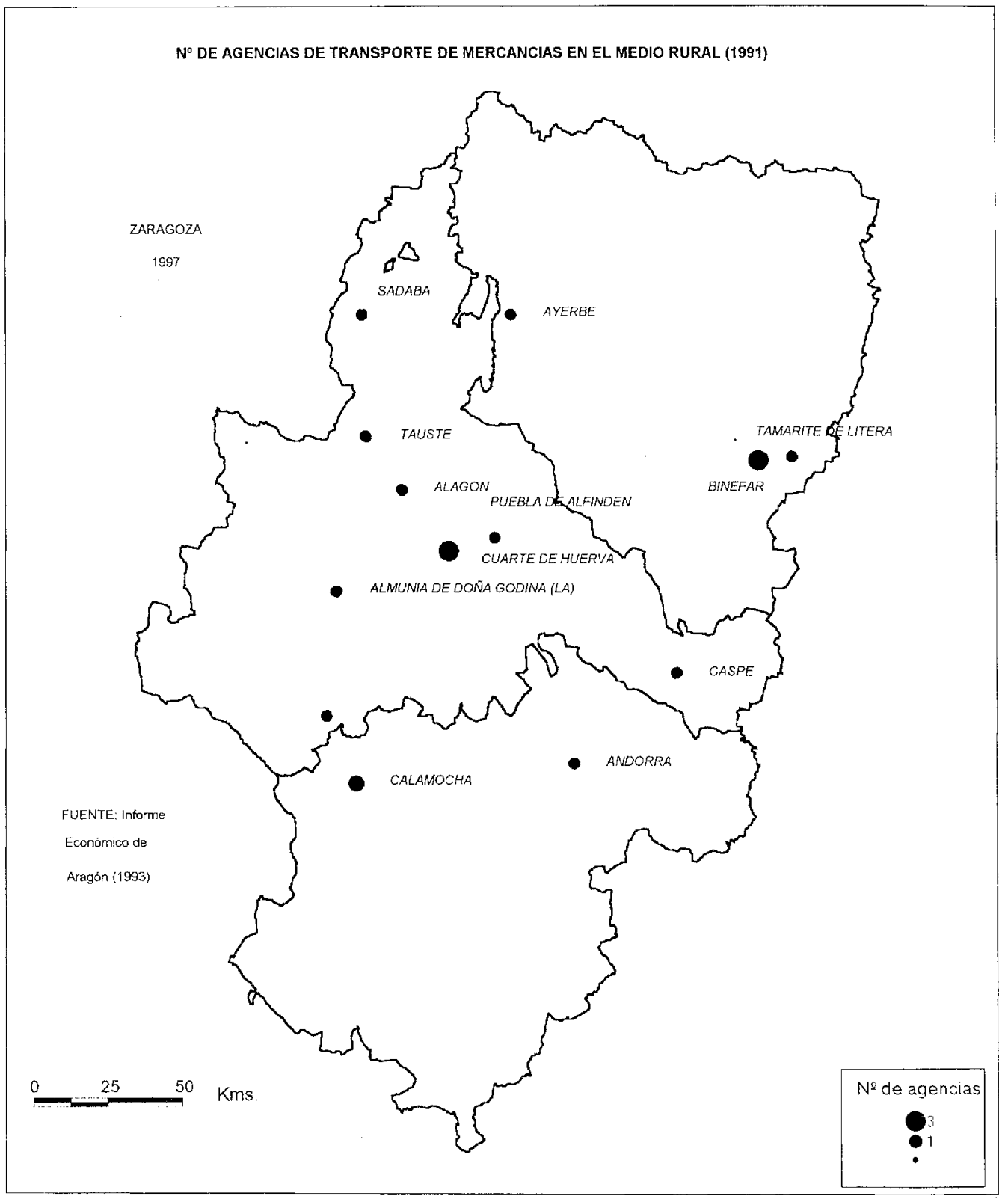


Por su parte, si observamos el mapa de localización empresarial vemos que enlos centros mencionados se concentra una parte considerable de las mismas. Así pues, parece lógico deducir que las empresas requieren servicios próximos a su localización capaces de gestionar las mercancías.

Con todo, la localización de empresas de transporte regular y de mercancías responden a las necesidades requeridas por la población trabajadora en las empresas y por la industria para el transporte de su producción. Ello sin olvidar la tendencia y posibilidades que ofrecen estos servicios en su medio tradicional como es la ciudad, que absorbe la mayor parte de ellos.

\section{CONSIDERACIONES FINALES}

Del análisis anterior se puede extraer que la mayor parte de la industria aragonesa se localiza en el medio rural, si bien la población prefiere residir en el medio urbano. Esa localización se concentra esencialmente en los corredores del Valle del Ebro y Jalón y en el entorno inmediato a Zaragoza, así como en los enclaves perifÉricos definidos por el río Cinca, el Bajo Aragón o los puntos aislados de Sabiñanigo y Calamocha.

Algo similar ocurre con la localización de los trabajadores en la industria, salvo la presencia de Opel España en Figueruelas que absorbe buena parte de los empleados rurales de la provincia de Zaragoza e induce puestos de trabajo en las empresas auxiliares ligadas a la multinacional.

Esta localización rural de la industria se correlaciona con servicios que requieren para su funcionamiento. Así pues, en las mismas sedes industriales o en sus proximidades se ubican diversas empresas de transporte regular para el traslado de trabajadores y agencias que se encargan de gestionar las mercancías producidas por las empresas.

\section{BIBLIOGRAFIA}

ALONSO LOGROÑO, M.P. (1993): El impacto de General Motors España. Estudio del medio rural circundante, Institución Fernando El Católico y Fundación Nueva Empresa, Zaragoza.

ALONSO LOGROÑO, M.P. (1996): "Localización de las industrias agroalimentarias en la provincia de Zaragoza", vil Cologuto de Geografía Rural, Jaca, Huesca. 
CALVO PALACIOS, J.L. (1994): "El Eje de desarrollo del Valle del Ebro y las perspectivas del Eje Norte-Sur", 3" Congreso de Economía Aragonesa, Zaragoza.

CLIMENT, E. y ALONSO, M.P. (1995): "El desarrollo industrial en Aragón, consecuencias de una situación estratégica", En BOSQUE, J. y MÉNDEZ, R. (Coords.): Cambio industrial y desarrollo regional en España, Oikos-Tau, Barcelona.

CONSEJO DE CAMARAS DE COMERCIO E INDUSTRIA (1993): Informe econónico de Aragón, Departamento de Economía e Industria, Zaragoza.

CUADRADO RIOURA J.R. (1992): "España en el marco económico y territorial europeo" En Ejes de desarrollo: España en la Europa de los noventa, Colegio de Economistas, Madrid.

D.G.A. (1997): Cuentas de la industria aragonesa. Año 1993, Instituto Aragonés de Estadística, Zaragoza.

D.G.A. (1994): Censo Industrial de Aragón 1993, Boletin de Indicadores Estadísticos de Aragón, 21, Instituto Aragonés de Estadística, Zaragoza.

HUERTA, E. (1992): "La industria" En SERRANO SANZ, M. (Dir.) y ot.: Estructura económica del Valle del Ebro, Espasa-Calpe, Madrid.

LABORIE, J.P. y LANGUMIER, J.E. (1982): "L'industrialización périurbaine", Reonc Géographique des Pyrénées et du Sud-Ouest, 53, 35-50.

MÉNDEZ, R. (Coord.) (1987a): Crecinniento industrial y descentralización production en los espacios periutrbanos de Madrid, Comunidad de Madrid, Madrid.

MOLINA, M. y MARÍN, J. (1981):" La industria", Geografía de Aragón, vol. 2, Guara editorial, Zaragoza.

MOLINERO, F. (1990): Los espacios rurales. Agricultura y sociedad en el mundo, Ariel Geografía, Barcelona. 